\title{
Gross tumor volume dependency on phase sorting methods of four-dimensional computed tomography images for lung cancer
}

\author{
Soo Yong Lee, MD, Sangwook Lim, PhD, Sun Young Ma, MD, PhD, Jesang Yu, MD \\ Department of Radiation Oncology, Kosin University Gospel Hospital, Kosin University College of Medicine, Busan, Korea
}

\begin{abstract}
Purpose: To see the gross tumor volume (GTV) dependency according to the phase selection and reconstruction methods, we measured and analyzed the changes of tumor volume and motion at each phase in 20 cases with lung cancer patients who underwent image-guided radiotherapy.

Materials and Methods: We retrospectively analyzed four-dimensional computed tomography (4D-CT) images in 20 cases of 19 patients who underwent image-guided radiotherapy. The 4D-CT images were reconstructed by the maximum intensity projection (MIP) and the minimum intensity projection (Min-IP) method after sorting phase as 40\%-60\%, 30\%-70\%, and 0\%-90\%. We analyzed the relationship between the range of motion and the change of GTV according to the reconstruction method.

Results: The motion ranges of GTVs are statistically significant only for the tumor motion in craniocaudal direction. The discrepancies of GTV volume and motion between MIP and Min-IP increased rapidly as the wider ranges of duty cycles are selected. Conclusion: As narrow as possible duty cycle such as 40\%-60\% and MIP reconstruction was suitable for lung cancer if the respiration was stable. Selecting the reconstruction methods and duty cycle is important for small size and for large motion range tumors.
\end{abstract}

Keywords: Four-dimensional computed tomography, Tumor volume, Respiratory-induced organ motion

\section{Introduction}

Intrafractional motion is an issue that is becoming increasingly important in the era of image-guided radiotherapy (IGRT). Intrafractional motion can be caused by the respiratory induced organ motion especially radiation therapy for lung cancer [1]. There are several methods to reduce the effect of respiratory motion in radiotherapy [2-9]. Most of these approaches, including four-dimensional (4D) imaging, 4D target delineation, increased planning margins, voluntary breath-hold and shallow breathing, abdominal compression, respiratory gating, and real-time tumor tracking have been used clinically in a variety of forms [2-9].

A promising solution for obtaining high quality computed tomography (CT) data in the presence of respiratory motion is 4D-CT [1]. Various 4D-CT based technologies have been proposed [5-8], and the maximum intensity projection (MIP) based internal target volume (ITV) technology is widely used due to the simple and rapid configuration of ITV based on a single $3 \mathrm{D}$ image. The MIP projection reflects the highest $\mathrm{CT}$ number encountered along the slice for each pixel of the volumetric data, displaying the brightest object along each

Received 07 September 2017, Revised 18 September 2017, Accepted 28 September 2017.

Correspondence: Sangwook Lim, PhD, Department of Radiation Oncology, Kosin University College of Medicine, 262 Gamcheon-ro, Seo-gu, Busan 49267, Korea. Tel: +82-51-990-6393, Fax: +82-303-3441-6247, E-mail: medicalphysics@hotmail.com

(c) This is an Open Access article distributed under the terms of the Creative Commons Attribution Non-Commercial License (http://creativecommons.org/ licenses/by-nc/4.0/) which permits unrestricted non-commercial use, distribution, and reproduction in any medium, provided the original work is properly cited.

www.e-roj.org 
slice at the maximum intensity during projection. Conversely, the minimum intensity projection (Min-IP) projection reflects the lowest CT number that occurs along the slice for each pixel in the volumetric data. MIP and Min-IP are standard volume rendering techniques used in diagnostic radiology [10-15].

The protocol for the gated radiation therapy of lung cancer patients in our department is that the phases selected from 4D-CT, as narrow as possible gating window such as 40\%-60\% phases are selected and reconstructed in MIP for minimizing the residual motion. In case of the patient's respiration is not stable, we select 30\%-70\% phases and set more margin for larger residual motion. For gated radiation therapy in our department, we obtain the setup images such as the fluorer images from On-Board Imager (OBI) at the end of exhalation of patient's breath. MIP reconstruction methods used for lung cancer and Min-IP used when the tumor to be treated was lower CT number than surrounding tissues such as liver cancers cases sometimes.

The aim of this study is to show the relationship between the reconstruction methods (MIP and Min-IP) and the target volumes, and also between the phase selections for reconstruction and the target volumes.

\section{Materials and Methods}

\section{Patients selection}

The 4D-CT images of lung cancer were retrospectively studied in this study. In order to see the GTV dependency, the combination of three phase (40\%-60\%, 30\%-70\%, and 0\%$90 \%$ ) selections which most frequently used phase selections and two reconstruction methods (MIP and Min-IP) applied to each 4D-CT case (Table 1).

Total 20 cases from lung cancer patients recently completed respiratory gated radiation therapy were selected. The cases could be roughly classified in four segments: right upper lobe $(R U L)$, right lower lobe (RLL), left upper lobe (LUL), and left lower lobe (LLL) (Table 2). These patients' 4D-CT images were sorted in MIP and already treated in 40\%-60\% duty cycle using Real-time Position Management (RPM; Varian Medical Systems Inc., Palo Alto, CA, USA) system.

\section{4D-CT technique}

The 4D-CT images were obtained using LightSpeed RT (GE Healthcare, Waukesha, WI, USA) with RPM system. We placed the infrared marker for RPM systems on the patients' xiphoid and asked the patients breathe freely and regularly. The 4D-CT images with phase information were sorted and reconstructed
Table 1. Six types of CT image reconstruction from 4D-CT images for a case

\begin{tabular}{|c|c|c|}
\hline Intensity projection & Phase selections & GTV \\
\hline \multirow[t]{3}{*}{ MIP } & $40 \%-60 \%$ & $\mathrm{GTV}_{\mathrm{MIP} 40 \%-60 \%}$ \\
\hline & $30 \%-70 \%$ & $\mathrm{GTV}_{\mathrm{MIP} 30 \%-70 \%}$ \\
\hline & $0 \%-90 \%$ & $\mathrm{GTV}_{\mathrm{MIPO} \%-90 \%}$ \\
\hline \multirow[t]{3}{*}{ Min-IP } & $40 \%-60 \%$ & $\mathrm{GTV}_{\mathrm{Min}-\mathrm{P} 40 \%-60 \%}$ \\
\hline & $30 \%-70 \%$ & $\mathrm{GTV}_{\text {Min-1P30\%-70\% }}$ \\
\hline & $0 \%-90 \%$ & $\mathrm{GTV}_{\mathrm{Min-1P0 \% -90 \%}}$ \\
\hline
\end{tabular}

4D-CT, four-dimensional computed tomography; MIP, maximum intensity projection; Min-IP, minimum intensity projection; GTV, gross tumor volume.

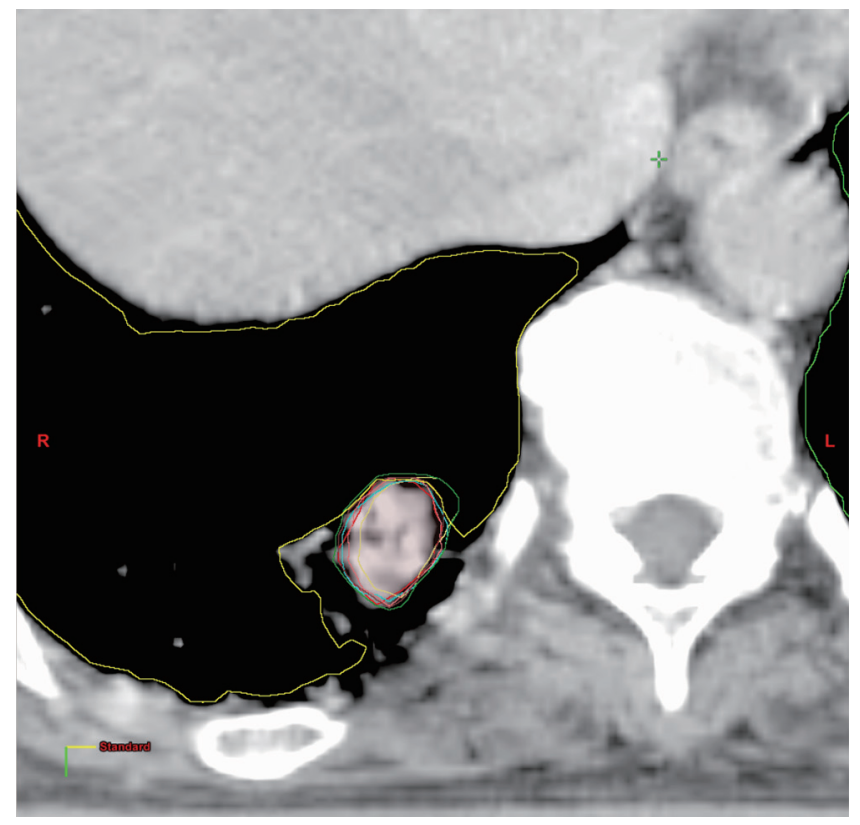

Fig. 1. Gross tumor volumes (GTVs) for 10 phases were delineated. The figure shows the overlapped GTVs of every phase on the same computed tomography images.

by Advantage Workstation (GE Healthcare).

Phases were sorted as 40\%-60\%, 30\%-70\%, and 0\%-90\% (all phases) from 4D-CT images and reconstructed as MIP and Min-IP methods, respectively. The phase of 40\%-60\% is routinely used to minimize the residual motion in our department and the phase of 30\%-70\% is used when the patients' respirations are not stable. Six types of reconstructed images were acquired for each case in Table 1.

\section{GTV delineation and phase selection}

As shown Fig. 1, the gross tumor volumes (GTV) were delineated on each slice of reconstructed CT image in the 
Table 2. GTV according to phase selection for 20 cases

\begin{tabular}{|c|c|c|c|c|c|c|c|}
\hline \multirow{2}{*}{$\begin{array}{l}\text { Case } \\
\text { no. }\end{array}$} & \multirow{2}{*}{ Sex } & \multirow{2}{*}{$\begin{array}{l}\text { Age } \\
(y r)\end{array}$} & \multirow{2}{*}{ Tumor location } & \multirow{2}{*}{$\begin{array}{c}\text { Motion range in } \\
\mathrm{RL} / \mathrm{AP} / \mathrm{CC} \text { directions }(\mathrm{mm})\end{array}$} & \multicolumn{3}{|c|}{ GTV/longest diameter $(\mathrm{mL} / \mathrm{mm})$} \\
\hline & & & & & MIP 40\%-60\% & MIP 30\%-70\% & MIP 0\%-90\% \\
\hline 1 & $\mathrm{~F}$ & 56 & - & $3.2 / 2.0 / 4.7$ & $3.70 / 22.8$ & $4.21 / 22.8$ & $5.70 / 25.2$ \\
\hline 2 & M & 61 & - & $3.1 / 19$ / 5.2 & 11.66 / 40.6 & $13.56 / 40.6$ & 16.53 / 43.8 \\
\hline 3 & M & 76 & RUL & $5.3 / 2.1 / 3.5$ & 19.43 / 38.3 & $20.36 / 37.9$ & 23.97 / 42.7 \\
\hline 4 & M & 84 & - & $0.7 / 2.3 / 1.0$ & 18.60 / 47.1 & $19.60 / 45.3$ & 19.80 / 47.0 \\
\hline 5 & $\mathrm{~F}$ & 47 & - & $0.9 / 1.3 / 2.1$ & 79.60 / 64.0 & 82.10 / 64.8 & 81.90 / 64.0 \\
\hline 6 & M & 71 & - & 0.7 / $1.3 / 12.1$ & 5.33 / 24.8 & 5.66 / 25.2 & 11.33 / 40.0 \\
\hline 7 & M & 55 & - & $1.0 / 3.3 / 11.0$ & 11.39 / 37.0 & 13.12 / 37.1 & 20.29 / 39.0 \\
\hline 8 & $\mathrm{~F}$ & 26 & RLL & $1.4 / 0.5 / 10.1$ & $11.31 / 37.4$ & 12.12 / 39.9 & 14.94 / 44.9 \\
\hline 9 & M & 61 & - & $2.1 / 3.7 / 19.1$ & 4.72 / 18.2 & 5.12 / 17.9 & 8.26 / 28.4 \\
\hline 10 & $M$ & 51 & - & 0.7 / 3.7 / 5.4 & $1.40 / 27.7$ & $1.90 / 27.7$ & $2.60 / 28.3$ \\
\hline 11 & $M$ & 61 & - & $1.1 / 3.7 / 6.5$ & 8.10 / 29.9 & 9.30 / 29.5 & 11.70 / 33.4 \\
\hline 12 & M & 70 & - & 0.8 / 1.9 / 3.2 & 45.90 / 51.0 & 49.10 / 51.0 & 52.90 / 51.3 \\
\hline 13 & M & 67 & LUL & $3.5 / 3.3$ / 4.8 & 8.70 / 29.9 & $10.40 / 30.6$ & 13.60 / 31.9 \\
\hline 14 & $\mathrm{~F}$ & 54 & - & $0.8 / 2.2$ / 3.7 & $4.20 / 20.4$ & $5.90 / 21.7$ & $6.40 / 22.1$ \\
\hline 15 & $\mathrm{~F}$ & 49 & - & $0.7 / 2.5 / 3.5$ & $1.30 / 15.1$ & $1.30 / 15.9$ & 1.70 / 17.1 \\
\hline 16 & $M$ & 61 & - & 4.3 / 2.8 / 9.5 & 0.87 / 13.5 & $0.98 / 13.4$ & $2.45 / 21.4$ \\
\hline 17 & $\mathrm{M}$ & 64 & - & $2.0 / 2.8 / 11.3$ & $2.03 / 14.8$ & 2.66 / 17.7 & 5.17 | 27.1 \\
\hline 18 & M & 51 & LLL & $0.6 / 1.0 / 6.5$ & $7.60 / 27.2$ & $8.00 / 27.8$ & $8.90 / 27.0$ \\
\hline 19 & $\mathrm{M}$ & 58 & - & 0.6 / 0.7 / 9.0 & $15.40 / 32.2$ & 16.30 / 32.0 & 21.30 / 36.5 \\
\hline 20 & $\mathrm{~F}$ & 60 & - & 1.4 / 1.3 / 9.0 & $4.00 / 21.4$ & 4.60 / 22.4 & $6.30 / 23.3$ \\
\hline
\end{tabular}

GTV, gross tumor volume; RL, right-left; $A$, anteroposterior; $C C$, craniocaudal; RLL, right lower lobe; LLL, left lower lobe; RUL, right upper lobe.

treatment planning system (Eclipse ver. 13.0, Varian Medical Systems Inc.) with lung window setting (a window width of $1500 \mathrm{HU}$, a window level of $-700 \mathrm{HU}$ ) through all cases by the same radiation oncologists. In this study, GTVs were named as follows, GTV 0 to $\mathrm{GTV}_{90}$ were contoured on every 10 phases in the 4D-CT. GTV MIP40\%-60\%, $_{\text {GTV }}$ MIP30\%-70\%, $_{\text {, }}$ and GTV $_{\text {MP00\%-90\% }}$ were contoured on MIP reconstructed $\mathrm{CT}$ images by selecting phases 40\%-60\%, 30\%-70\%, and 0\%-90\%, respectively. GTV 1P40\%-60\%, $\mathrm{GTV}_{\text {Min-1P30\%-70\%, }}$ and $\mathrm{GTV}_{\text {Min-1P0\%-90\% }}$ were contoured on Min-IP images in the same manner.

\section{Data analysis}

In order to see the trajectory of moving GTVs, GTVs' center of mass in right-left (RL), anteroposterior (AP), and craniocaudal (CC) direction were measured for 10 phases in treatment planning system. The motion ranges in $3 \mathrm{D}$ were measured for each case. The relationship between the motion ranges and the variation of GTV according to the reconstruction methods were analyzed.

\section{Results}

\section{GTV motion measurements}

The motion ranges of the tumors in lung for 20 cases were measured 3-dimensionally in Table 2. Fig. 2 shows the motion ranges for each segment of lung as box-and-whisker plots. The margin of the box means 25\% from average of Gaussian distribution. The horizontal bar in the center of the box means average and the upper and lower horizontal bars are for the maximum and the minimum motion ranges of the 20 cases. The maximum GTV motion range is about $2 \mathrm{~cm}$. The motion ranges for 4 segments of lung (RUL, RLL, LUL, and LLL) were measured, respectively in the Fig. 3 . The average motion ranges were $1.73 \mathrm{~mm}, 3.52 \mathrm{~mm}$, and $6.39 \mathrm{~mm}$ for $R L, A P$, and CC directions. The results of the motion ranges dependency according to tumor location are shown in Table 3. The tumor motion in RL direction was not shown in Table 3, since RL motion ranges were not normally distributed. The motion ranges of GTVs are statistically significant only for the tumor motion in craniocaudal direction. The average tumor motion range of $10.30 \mathrm{~mm}$ in the lower lobe group (RLL and LLL) was statistically greater than the average of $3.82 \mathrm{~mm}$ in the upper 


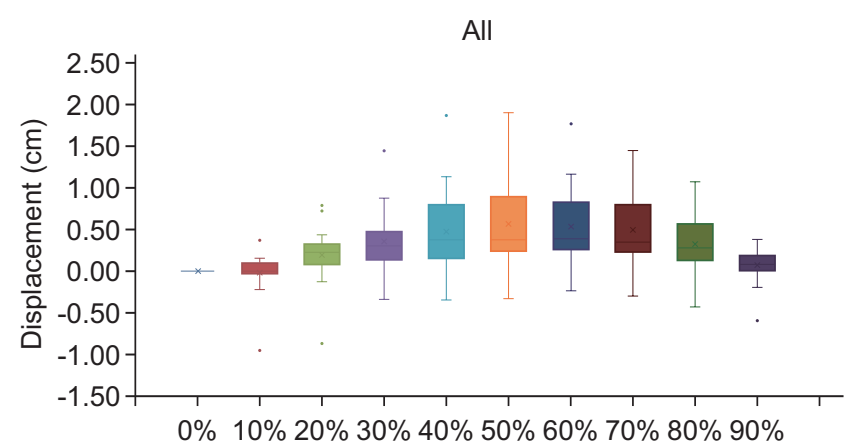

RUL
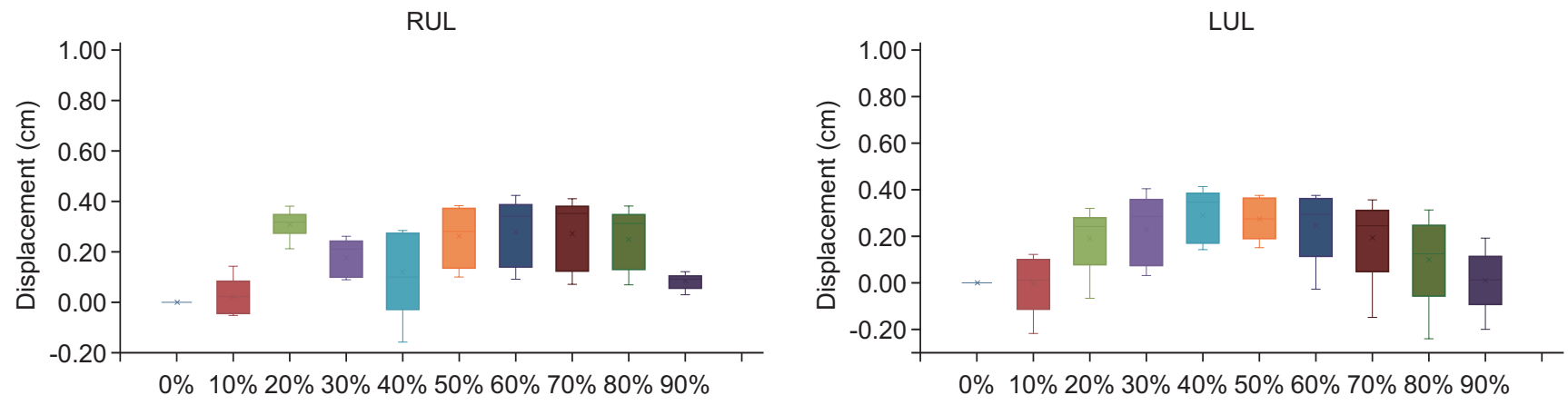

RLL


Fig. 2. The motion (in $\mathrm{cm}$ ) for 20 cases of lung cancer were classified in four segments-right upper lobe (RUL), right lower lobe (RLL), left upper lobe (LUL), and left lower lobe (LLL)-as box-and-whisker plot. The tumor with the largest motion range was in RLL.

lobe group (RUL and LUL). The average motion ranges were 3.30 $\mathrm{mm}, 11.54 \mathrm{~mm}, 4.34 \mathrm{~mm}$, and $9.06 \mathrm{~mm}$ for RUL, RLL, LUL, and LLL, respectively (Table 2).

\section{GTV dependency on phase selection and reconstruction methods}

Fig. 3 shows example of the same case for lung cancer. The GTV varies according to the phase selections and sorting methods. The GTVs were varied according to the phase selections in Table 2. The largest GTV was in case \#5 and the smallest GTV was in case \#16.

Fig. 4 shows the discrepancy of the GTVs for 20 cases of lung cancer according to the phase selections and the reconstruction methods. Fig. 4A shows the GTV dependency on phase selections in MIP sorting. Fig. $4 \mathrm{~B}$ shows the
Table 3. Motion range of GTV in craniocaudal direction found to be significant at lower lobe of the lung

\begin{tabular}{lcc}
\hline \multicolumn{1}{c}{ Location } & AP $(\mathrm{mm})$ & $\mathrm{CC}(\mathrm{mm})$ \\
\hline Upper lobe (RUL, LUL) & $2.32 \pm 0.70$ & $3.82 \pm 1.58$ \\
Lower lobe (RLL, LLL) & $2.11 \pm 1.27$ & $10.30 \pm 3.72$ \\
t-value & 0.457 & -5.070 \\
p-value & 0.655 & 0.000 \\
\hline
\end{tabular}

Values are presented as mean \pm SEM.

GTV, gross tumor volume; AP, anteroposterior; CC, craniocaudal; RUL, right upper lobe; LUL, left upper lobe; RLL, right lower lobe; LLL, left lower lobe; SEM, standard error of the mean.

GTV variation on phase selections in Min-IP sorting. The discrepancies increased rapidly when the larger ranges of phases are selected. 


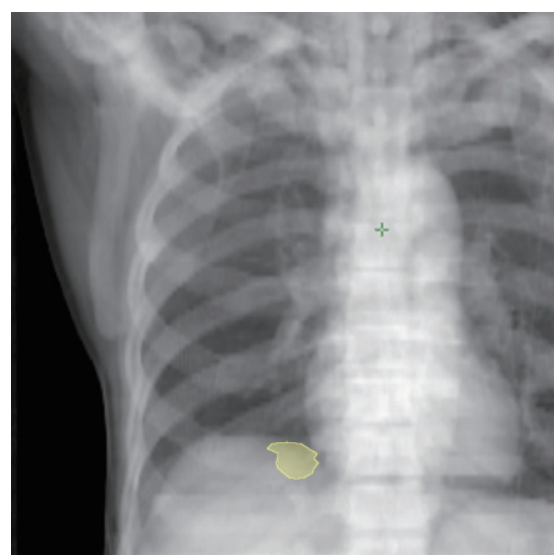

GTV $_{\text {MIP } 40 \%-60 \%}: 4.72 \mathrm{~mL}$

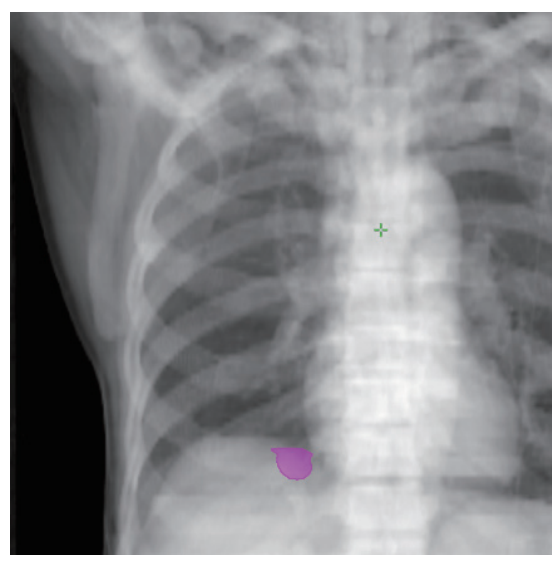

GTV $_{\text {Min-IP40\%-60\% }}: 3.14 \mathrm{~mL}$



$\mathrm{GTV}_{\text {MIP } 30 \%-70 \%}: 5.12 \mathrm{~mL}$

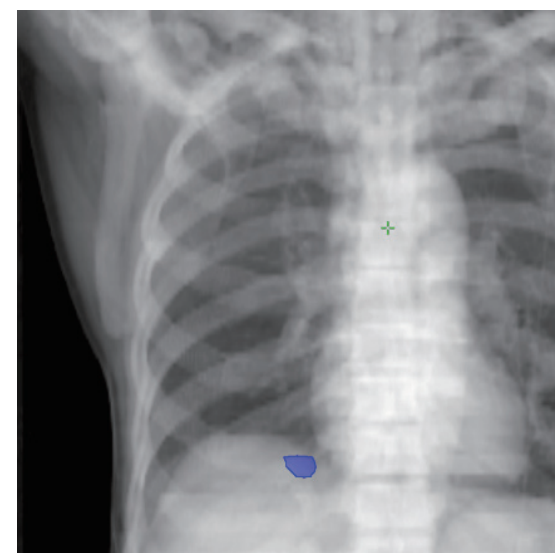

GTV $_{\text {Min-IP30\%-70\% }}: 1.50 \mathrm{~mL}$

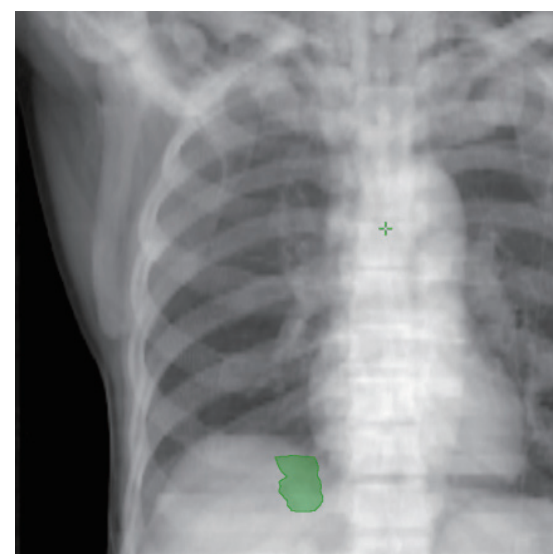

$\mathrm{GTV}_{\mathrm{MIP0} \%-90 \%}: 8.26 \mathrm{~mL}$

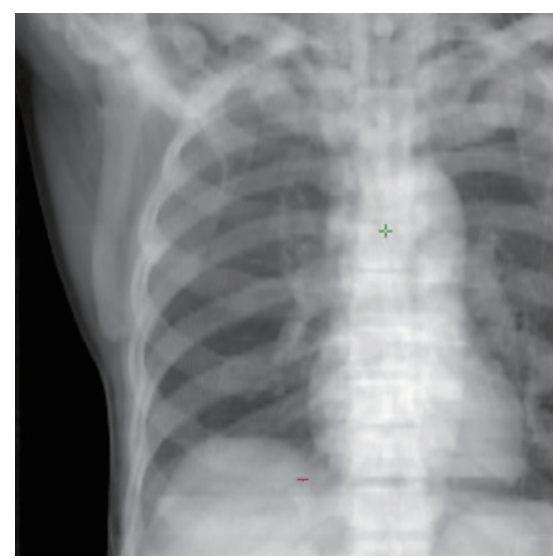

$\mathrm{GTV}_{\text {Min-IP0\%-90\% }}: 0.01 \mathrm{~mL}$

Fig. 3. One of cases of gross tumor volume (GTV) dependency according to the phase selection and the sorting methods. MIP, maximum intensity projection; Min-IP, minimum intensity projection.
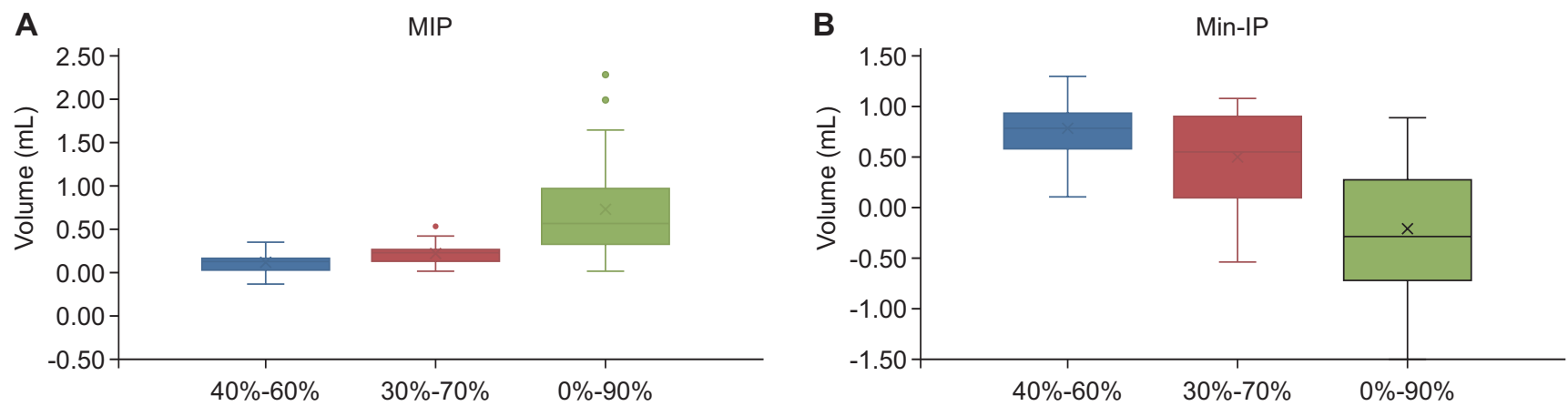

Fig. 4. The variation of the gross tumor volumes (GTVS) for 20 cases of lung cancer according to the phase selections and the reconstruction methods as a box-and-whisker plot. (A) Maximum intensity projection (MIP) reconstruction. (B) Minimum intensity projection (Min-IP) reconstruction.

\section{Discussion and Conclusion}

The target motions in the lung are dominant in craniocaudal direction. Davies et al. [16] and Korin et al. [17] found that upper abdominal organ motion in the anterior/posterior and right/left directions are less $2 \mathrm{~mm}$ displacement. The tumor 
motions in the lower lung were observed larger than upper lung as study of Brandner et al. [18]. We had to compromise between the residual motion and the treatment time.

We set the duty cycle usually around 50\% phase since normally $50 \%$ phase is at full exhalation which is relatively stable. The discrepancies between MIP and Min-IP increased rapidly as the wider ranges of duty cycles are selected. In case of the respiration regularity is poor to select 40\%-60\% dusty cycle, 30\%-70\% duty cycle can be selected and the PTV margin enlarged. Most of CT reconstruction software can only divide 10 phases for patient's respiration.

Muirhead et al. [8] found that MIP-based ITV technique is safe, but may not be the case when the tumor is at or near the diaphragm. That protocol will underestimate actual ITV. This occurs because the electron density resembles the tumor in the diaphragm and other surrounding healthy tissue. It is because once the MIP is generated it cannot distinguish the boundary between the density environment and the overlapping tumor. Therefore, this protocol should not be commissioned in all situations.

When GTV was delineated based on the CT images from 4D$\mathrm{CT}$, the volume depended on the motion ranges of the tumor's center of mass and the reconstruction methods. As narrow as possible duty cycle such as 40\%-60\% and MIP reconstruction was suitable for lung cancer if the respiration was stable. MinIP reconstruction could underestimate the GTV for lung cancer. Incorrect selection of the reconstruction methods could result in significant targeting error. Selecting the reconstruction methods such as MIP or Min-IP is important for small size and for large motion range tumors.

\section{Conflict of Interest}

No potential conflict of interest relevant to this article was reported.

\section{Acknowledgments}

This research was supported by Basic Science Research Program through the National Research Foundation of Korea (NRF) funded by the Ministry of Education, Science and Technology (No. 2010-0013701 and 2013R1A1A2012013).

\section{References}

1. Keall PJ, Mageras GS, Balter JM, et al. The management of respiratory motion in radiation oncology report of AAPM Task
Group 76. Med Phys 2006;33:3874-900.

2. Lim S, Park SH, Ahn SD, et al. Guiding curve based on the normal breathing as monitored by thermocouple for regular breathing. Med Phys 2007;34:4514-8.

3. Lagerwaard FJ, Van Sornsen de Koste JR, Nijssen-Visser MR, et al. Multiple "slow" CT scans for incorporating lung tumor mobility in radiotherapy planning. Int J Radiat Oncol Biol Phys 2001;51:932-7.

4. Wong JW, Sharpe MB, Jaffray DA, et al. The use of active breathing control $(A B C)$ to reduce margin for breathing motion. Int J Radiat Oncol Biol Phys 1999;44:911-9.

5. Rietzel E, Liu AK, Doppke KP, et al. Design of 4D treatment planning target volumes. Int J Radiat Oncol Biol Phys 2006;66:287-95.

6. Lim S, Ahn SD, Park SH, et al. Study of respiration simulating phantom using thermocouple-based respiration monitoring mask. J Korean Soc Ther Radiol Oncol 2005;23:217-22.

7. Underberg RW, Lagerwaard FJ, Slotman BJ, Cuijpers JP, Senan S. Use of maximum intensity projections (MIP) for target volume generation in 4DCT scans for lung cancer. Int J Radiat Oncol Biol Phys 2005;63:253-60.

8. Muirhead R, McNee SG, Featherstone C, Moore K, Muscat S. Use of Maximum Intensity Projections (MIPs) for target outlining in 4DCT radiotherapy planning. J Thorac Oncol 2008;3:1433-8.

9. Wolthaus JW, Sonke JJ, van Herk M, et al. Comparison of different strategies to use four-dimensional computed tomography in treatment planning for lung cancer patients. Int J Radiat Oncol Biol Phys 2008;70:1229-38.

10. Cody DD. AAPM/RSNA physics tutorial for residents: topics in CT image processing in CT. Radiographics 2002;22:1255-68.

11. Gruden JF, Ouanounou S, Tigges S, Norris SD, Klausner TS. Incremental benefit of maximum-intensity-projection images on observer detection of small pulmonary nodules revealed by multidetector CT. AJR Am J Roentgenol 2002;179:149-57.

12. Persson A, Dahlstrom N, Engellau L, Larsson EM, Brismar $T B$, Smedby 0 . Volume rendering compared with maximum intensity projection for magnetic resonance angiography measurements of the abdominal aorta. Acta Radiol 2004;45:453-9.

13. Jeong YJ, Lee KS, Yoon YC, Kim TS, Chung MJ, Kim S. Evaluation of small pulmonary arteries by 16 -slice multidetector computed tomography: optimum slab thickness in condensing transaxial images converted into maximum intensity projection images. J Comput Assist Tomogr 2004;28:195-203.

14. van Straten $M$, Venema HW, Streekstra GJ, Majoie CB, 
den Heeten GJ, Grimbergen CA. Removal of bone in CT angiography of the cervical arteries by piecewise matched mask bone elimination. Med Phys 2004;31:2924-33.

15. Aziz ZA, Padley SP, Hansell DM. CT techniques for imaging the lung: recommendations for multislice and single slice computed tomography. Eur J Radiol 2004;52:119-36.

16. Davies SC, Hill AL, Holmes RB, Halliwell $M$, Jackson PC. Ultrasound quantitation of respiratory organ motion in the upper abdomen. Br J Radiol 1994;67:1096-102.
17. Korin HW, Ehman RL, Riederer SJ, Felmlee JP, Grimm RC. Respiratory kinematics of the upper abdominal organs: a quantitative study. Magn Reson Med 1992;23:172-8.

18. Brandner ED, Chetty IJ, Giaddui TG, Xiao Y, Huq MS. Motion management strategies and technical issues associated with stereotactic body radiotherapy of thoracic and upper abdominal tumors: a review from NRG oncology. Med Phys 2017;44:2595-612. 